\title{
Bestimmung des Sauerstoffs im venösen Blute bei einigen chirurgischen Erkrankungen, mit besonderer $\mathrm{Be}=$ rïcksichtigung bösartiger Geschwïlste.
}

\author{
Von \\ Yoshio Kitagawa. \\ (北川芳夫) \\ (Aus der Chinurgischen Klinik der Universität Sendai. \\ Direktor: Prof. Dr. Sh. Sugimura)
}

Im Jahre 1929 bestimmte Sehrt kolorimetrisch den Sauerstoff des Bluts bei Krebskranken und Krebsdisponierten durch die Indophenolblaureaktion. Bei 33 sogenannten normalen und auch bei Fällen mit unbedeutenden Erkrankungen betrug der Durchschnitt der von ihm sogenannten Differenzwerte 17,73, wogegen der Durchschnittsdifferenzwert 8 sicherer Krebsfälle 8,5 war. Diese neue Methode scheint nach dem Autor vor allem einen bemerkenswerten Einblick in die Bluteigenart des an Krebs Erkrankten und des zu dieser Krankheit Disponierten zu bieten. Über die Bestimmung der Blutgase und der mit ihnen in engerer Beziehung stehenden Faktoren bei chirurgischen Erkrankungen, vor allem auch bei malignen Geschwülsten gibt es bisher nur wenige Veröffentlichungen. So bestimmten Moore u. Wilson, Posharskaja und Menton die Alkalität, Porges u. Leimdörfer die alveoläre Kohlensäurespannung, Roffo u. Correa das $p H$, Luttinger und Sannié u. Peyre die Alkalireserve und das $p \mathrm{H}$, Vana das Säure-Basengleichgewicht und Sejhar, das $p \mathrm{H}$, die Alkalireserve und die alveoläre Kohlensäurespannung des Bluts bei malignen Geschwülsten. Auch T. Ka to bestimmte den Kohlensäuregehalt und die Alkalitoleranz des Blutplasmas bei Magenkrebsen und Uno das Kohlensäurebindungsvermögen des Bluts bei verschiedenen chirurgischen Erkrankungen und zwar bei malignen Geschwülsten.

Da die Kenntnis des Verhaltens der Blutgase, vor allem des Sauerstoffs im Blute bei chirurgischen Erkrankungen, besonders auch bei 
malignen Geschwülsten, noch ziemlich lückenhaft zu sein und die Ansicht von Sehrt noch einer Nachprüfung, auch auf anderem Wege, zu bedürfen scheint, möchte ich im folgenden die Ergebnisse meiner Untersuchung des Sauerstoffgehalts des venösen Bluts bei einigen chirurgischen Erkrankungen, besonders bei malignen Geschwülsten kurz mitteilen.

\section{Meine Untersuchungsmethode.}

Ich untersuchte mit dem Barcroft schen Differentialmanometer (Makroapparat) die Sauerstoffunsättigung und -kapazität und die prozentuelle Sauerstoffsättigung des venösen Bluts unter bestimmten Bedingungen bei 183 Fällen, bei denen 209 Untersuchungen vorgenommen wurden. Darunter befanden sich 39 Gesunde, 51 maligne Tumoren, 38 Fälle chirurgischer Nierenerkrankungen, mit Ausschluss von Geschwülsten, und 55 Fälle verschiedener sonstiger chirurgischer Erkrankungen, vor allem auch akuter Infektionen. Bei allen diesen Fällen wurden daneben auch Pulsfrequenz, Atmungszahl, Körpertemperatur, Blutkörperchenzahl, Hümoglobingehalt des Bluts, etwa vorhandene hereditäre Belastung beim Krebs und beim Magenkrebs auch der Magensaft untersucht und bei chirurgischen Nierenerkrankungen die Nierenfunktion geprüft und diese Ergebnisse berücksichtigt.

Da bei der Blutgasbestimmung, besonders des venösen Bluts, wie schon $\nabla$ on mehreren Autoren hervorgehoben, als Vorbedingungen verschiedene äussere Faktoren, individuelle Verschiedenheiten und zeitliche Schwankungen berücksichtigt werden muissen und der Sauerstoffgehalt des Bluts nach Su ga n u ma in der Nacht sinkt und am frühen Morgen steigt, so ist es nötig, die Untersuchungen unter möglichst gleichmüssig bestimmten Bedingungen auszuführen. Bei meinen Untersuchungen wurden alle Fälle mit Fieberanstieg, Veränderungen des Pulses, Atembeschwerden oder Menstruation ausgeschlossen. Die Untersuchung wurde stets am frühen Morgen in nüchternem Zustande und nach mindestens 30 Minuten langer Bettruhe und unter Losmachen der Kleidung ausgeführt, um jeden Einfluss durch Nahrungsaufnahme und Körperbewegung auszuschalten. Einnehmen von Arzneimitteln wurde anch schon für den Tag vor der Untersuchung verboten. Nur wenige akute, bedrohliche Kranke wie Ileus, Appendizitis und Perforationsperitonitis, die unmittelbar vor dringender Notoperation untersucht werden mussten, bildeten Ausnahmen.

Das Blut wurde durch Punktion der Vena mediana in der Ellbogengrube ohne Drücken, mit einer Rekordspritze von $2 \mathrm{ccm}$ Inhalt nach Anfeuchten des Nadelhohlraums mit einer 2\%igen Lösung von Natrium citricum entnommen. Das so entnommene Blut wurde sofort, ohne in Berührung mit der Luft zu kommen, ius Barcroftsche Differentialmanometer eingesogen und dann seine Saverstoffunsättigung und die Sauerstoffkapazität, somit auch die prozentuelle Sauerstoffsättigung bestimmt. Für diese Bestimmungen verwendete ich, wie erwähnt, die Barcroftsche Methode mit dem Differentialmanometer (Makroapparat). Dabei bestimmte ich die Konstante des Apparats nach der Hof fmann- 
schen Methode. Für die technischen Einzelheiten bei der Ausführung der Methode sei auf Barcroft und Straub verwiesen.

\section{Ergebnisse meiner Untersuchungen.}

Meine Untersuchungen bei Gesunden umfassten 39 Personen verschiedenen Alters, die nach klinischer Untersuchung keine Besonderheiten, vor allem nicht an Herz, Lungen, Verdauungsorganen und Nieren noch auch im Blut aufwiesen. Davon waren 24 Männer und 15 Frauen. Die Ergebnisse meiner Untersuchungen Gesunder sind übersichtlich in Tabelle I wiedergegeben.

Über die Blutgasbestimmung venösen Bluts Gesunder liegen schon mehrere Berichte vor. In den Zahlen der Sauerstoffunsättigung des venösen Bluts Gesunder scheinen, wie Tabelle II zeigt, die Ergebnisse der Autoren weit auseinanderzugehen. Nach T. Kato verhält sich die Sauerstoffunsättigung des venösen Bluts je nach der Punktionsstelle und der Bestimmungsweise verschieden, beträgt aber bei Gesunden, und zwar in Ruhe, durchschnittlich 4 bis $6 \mathrm{ccm}$ in $100 \mathrm{ccm}$ Blut. Aus Tabelle II lässt sich wohl annehmen, dass, mit Ausnabme von Suganuma und Tanaka, die Zahlen japanischer Forscher über die Sauerstoffunsättigung venösen Bluts gesunder Japaner um annähernd $10 \mathrm{ccm}$ liegen und deshalb diese Zahl als der ungefähre Durchschnittswert betrachtet werden darf. Nach meinem Ergebnis beträgt die Sauerstoffunsättigung venösen Bluts Gesunder zwischen 15,86 und $6,27 \mathrm{ccm}$, durchschnittlich $9,08 \mathrm{ccm}$ in $100 \mathrm{ccm}$ Blut bei $0^{\circ} \mathrm{C}, 760 \mathrm{~mm}$ $\mathrm{Hg}$.

Mein Ergebnis bei der Sauerstoffkapazität venösen Bluts Gesun-

$$
\text { Tabelle I. }
$$

Untersuchungen bei Gesunden.

\begin{tabular}{|c|c|c|c|c|}
\hline Geschlecht & & $\begin{array}{l}\text { Sauerstoff- } \\
\text { unsättigung } \\
\text { (ccm) }\end{array}$ & $\begin{array}{c}\text { Sauerstoff- } \\
\text { kapazität } \\
\text { (ccm) }\end{array}$ & $\begin{array}{c}\text { Prozentuelle } \\
\mathrm{O}_{2} \text {-Sïttigung } \\
(\%)\end{array}$ \\
\hline Männlich & $\begin{array}{l}\text { Maximum } \\
\text { Minimum } \\
\text { Durchschnitt }\end{array}$ & $\begin{array}{r}15,86 \\
6,52 \\
9,23\end{array}$ & $\begin{array}{l}22,44 \\
16,40 \\
20,51\end{array}$ & $\begin{array}{l}69,5 \\
29,3 \\
54,9\end{array}$ \\
\hline \multirow[t]{2}{*}{ Weiblich } & $\begin{array}{l}\text { Maximum } \\
\text { Minimum } \\
\text { Durchschnitt }\end{array}$ & $\begin{array}{r}12,61 \\
6,27 \\
8,77\end{array}$ & $\begin{array}{l}20,74 \\
16,84 \\
18,13\end{array}$ & $\begin{array}{l}65,8 \\
36,1 \\
51,6\end{array}$ \\
\hline & Durchschnitt & 9,08 & 19,59 & 53,6 \\
\hline
\end{tabular}


Tabelle $\Pi$.

Sauerstoffunsättigung des venösen Bluts bei Gesunden.

\begin{tabular}{|c|c|c|c|c|c|}
\hline \multirow{2}{*}{ Autoren } & \multirow{2}{*}{$\begin{array}{l}\text { Zahl d. } \\
\text { Unter- } \\
\text { suchten }\end{array}$} & \multicolumn{3}{|c|}{ Sauerstoffunsättigung Vol. $\%$} & \multirow{2}{*}{$\begin{array}{c}\text { Bestimmungs- } \\
\text { methode }\end{array}$} \\
\hline & & Mraximum & Minimnm & $\begin{array}{l}\text { Durch- } \\
\text { schnitt }\end{array}$ & \\
\hline$\underset{(1918)}{\operatorname{Lundsg} a r d}$ & 12 & 8,95 & 2,70 & 5,80 & Van Slyke \\
\hline $\begin{array}{l}\text { Stadie } \\
\qquad(1919)\end{array}$ & 5 & 6,30 & 4,90 & 5,60 & $"$ \\
\hline $\begin{array}{c}\text { Sugan uma } \\
(1920)\end{array}$ & $10\left\{\begin{array}{l}5 \mathrm{~m} \\
5 \mathrm{w}\end{array}\right.$ & $\begin{array}{l}3,38 \\
-\end{array}$ & $\underline{1,94}$ & $\begin{array}{l}2,90 \\
4,32\end{array}$ & Plesch \\
\hline $\begin{array}{r}\text { Tanaka } \\
(1922)\end{array}$ & 11 & - & 一 & 5,70 & Barcroft \\
\hline $\begin{array}{l}\text { Odaila } \\
\qquad(1924)\end{array}$ & 37 & 23,99 & 5,55 & 11,45 & " \\
\hline $\begin{array}{l}\text { K imura u. } \\
\text { Yamada (1928) }\end{array}$ & $13\left\{\begin{array}{l}8 \mathrm{~m} \\
5 \mathrm{w}\end{array}\right.$ & $\begin{array}{l}16,00 \\
12,10\end{array}$ & $\begin{array}{r}8,80 \\
10,00\end{array}$ & $\begin{array}{l}13,80 \\
11,60\end{array}$ & $"$ \\
\hline Verfasser & $39\left\{\begin{array}{l}24 \mathrm{~m} \\
15 \mathrm{w}\end{array}\right.$ & $\begin{array}{l}15,86 \\
12,61\end{array}$ & $\begin{array}{l}6,52 \\
6,27\end{array}$ & $\begin{array}{l}9,23 \\
8,77\end{array}$ & $"$ \\
\hline
\end{tabular}

der beträgt zwischen 22,44 und $16,40 \mathrm{ccm}$, durchschnittlich $19,59 \mathrm{ccm}$ bei $0^{\circ} \mathrm{C}, 760 \mathrm{~mm} \mathrm{Hg}$ (siehe Tabelle I). Wenn ich diese meine Zahlen mit denen der anderen Forscher, mit Ausschluss von Suganuma, vergleiche (siehe Tabelle III), so ergibt sich, dass die Zahlen im grossen und ganzen miteinander übereinstimmen und ihr Durchschnittswert ungefähr $20 \mathrm{~cm}$ beträgt. Ferner sei hier bemerkt, dass die Sauerstoffunsättigung und -kapazität venösen Bluts Gesunder beim männlichen Geschlecht durchschnittlich grösser als beim weiblichen sind. So betrug der Durchschnittswert der Sauerstoffunsättigung beim männlichen Geschlecht $9,23 \mathrm{ccm}$, beim weiblichen $8,77 \mathrm{ccm}$ und der der Sauerstoffkapazität beim männlichen $20,51 \mathrm{ccm}$, beim weiblichen 18,13 ccm (siehe Tabellen I, II u. III).

Die prozentuelle Sauerstoffsättigung venösen Bluts Gesunder beträgt nach Tanak a von 63,4 bis $76,1 \%$, durchschnittlich $69,7 \%$. Meine Zahlen lagen zwischen $69,5 \%$ und $29,3 \%$, betrugen durchschnittlich 53,6\%, liegen also etwas niedriger als die anderer Autoren.

Beim malignen Geschwülsten beobachteten Autoren wie Mo ore u. Wilson, Menton, Burrows, Roffou. Correa, Sanniéu.Peyre, Vana, Sejhar, Weiss, Sümegi u. v. Udvardy Zunahme der Alka- 
Tabelle III.

Sauerstoff kapazität des venösen Bluts bei Gesunden.

\begin{tabular}{|c|c|c|c|c|c|}
\hline \multirow{2}{*}{ Autoren } & \multirow{2}{*}{$\begin{array}{l}\text { Zahl d. } \\
\text { Unter- } \\
\text { suchten }\end{array}$} & \multicolumn{3}{|c|}{ Sauerstoffkapazität Vol. $\%$} & \multirow{2}{*}{$\begin{array}{l}\text { Bestimmungs- } \\
\text { methode }\end{array}$} \\
\hline & & Maximum & Minimum & $\begin{array}{l}\text { Durch- } \\
\text { schnitt }\end{array}$ & \\
\hline $\begin{array}{l}\text { Haldane u. } \\
\text { Smith }(1900)\end{array}$ & 14 & 20,90 & 16,00 & 18,50 & Ferricyankali \\
\hline Lundsga ard & 12 & 26,90 & 12,30 & 19,50 & Van Slyke \\
\hline $\begin{array}{l}\text { Stadie } \\
\qquad(1919)\end{array}$ & 5 & 23,30 & 19,10 & 21,20 & $"$ \\
\hline $\begin{array}{l}\text { H arrop } \\
(1919)\end{array}$ & 15 & 24,70 & 14,40 & 20,10 & $"$ \\
\hline$\underset{(1919)}{\text { Suga a ra }}$ & 13 & 23,20 & 17,30 & 20,30 & Barcroft \\
\hline $\begin{array}{c}\text { Suganuma } \\
(1920)\end{array}$ & $10\left\{\begin{array}{l}5 \mathrm{~m} \\
5 \mathrm{w}\end{array}\right.$ & $\underline{15,74}$ & 13,90 & $\begin{array}{l}14,48 \\
12,01\end{array}$ & Plesch \\
\hline $\begin{array}{r}\text { Tanaka } \\
(1922)\end{array}$ & 11 & 23,60 & 16,30 & 18,60 & Barcroft \\
\hline $\begin{array}{c}\text { Yamauchi } \\
(1928)\end{array}$ & $8\left\{\begin{array}{l}m \\
w\end{array}\right.$ & $\begin{array}{l}20,40 \\
19,30\end{array}$ & $\begin{array}{l}18,30 \\
18,00\end{array}$ & $\begin{array}{l}19,10 \\
18,40\end{array}$ & $"$ \\
\hline $\begin{array}{l}\text { Kimura u. } \\
\text { Y a mad a (1928) }\end{array}$ & $16\left\{\begin{array}{l}8 \mathrm{~m} \\
8 \mathrm{w}\end{array}\right.$ & $\begin{array}{l}22,00 \\
21,00\end{array}$ & $\begin{array}{l}19,00 \\
15,60\end{array}$ & $\begin{array}{l}20,90 \\
18,00\end{array}$ & $"$ \\
\hline Verfasser & $39\left\{\begin{array}{l}24 \mathrm{~m} \\
15 \mathrm{w}\end{array}\right.$ & $\begin{array}{l}22,44 \\
20,74\end{array}$ & $\begin{array}{l}16,40 \\
16,84\end{array}$ & $\begin{array}{l}20,51 \\
18,13\end{array}$ & $"$ \\
\hline
\end{tabular}

lität des Bluts und Autoren wie Posharskaja, T. Kato und Uno Azidosis. Sehrt stellte, wie erwähnt, bei Krebskranken mit seiner Indophenolblaureaktion Abnahme des Sauerstoffgehalts des Bluts fest.

Ich habe den Sauerstoff im venösen Blut bei 51 Fällen maligner Tumoren mit verschiedener Lokalisation und verschiedenem Wachstumszustand unter den oben angeführten Bedingungen quantitativ bestimmt. Darunter gab es 44 Karzinome und 7 Sarkome. Beim Krebs betrafen 16 den Magen, 6 das Rektum, bei 4 lag Karzinom des Urogenitalorgans vor, bei 10 das des Gesichts und Halses einschliesslich der Zunge, bei 5 das der Brustdrüse und bei 3 das der Extremitätenweichteile. Meine Ergebnisse sind in Tabelle IV wiedergegeben (siehe Tabelle IV).

Aus diesen Ergebnissen geht hervor, dass die Sauerstoffunsätti- 
Tabelle IV.

Untersuchungen bei Karzinomen und Sarkomen.

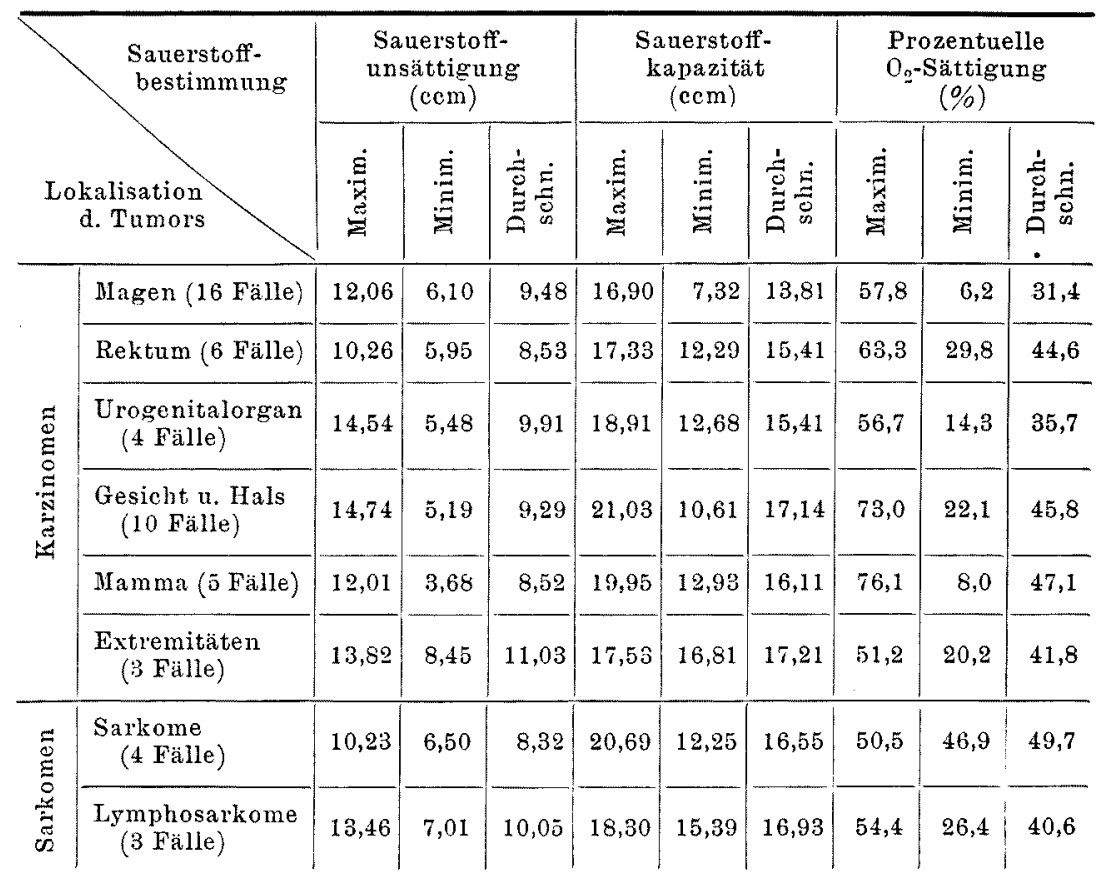

gung venösen Bluts bei Karzinomen im grossen und ganzen anrähernd normale, bei Extremitätenkrebsen sogar nur etwas erhöhte Werte zeigte. Die Sauerstoffkapazität venösen Bluts war bei Karzinomen des Magens erheblich niedriger als die Norm, so dass die prozentuelle Sauerstoffsättigung dabei deutlich vermindert erschien. Bei Karzinomen des Rektums und des Urogenitaltraktus hatte die Sauerstoffkapazität ein wenig, bei denen des Gesichts und des Halses sowie der Brustdrise nur etwas abgenommen. Bei Extremitätenkrebsen zeigte die Sauerstoffkapazität fast normale Werte. Die prozentuelle Sauerstoffsättigung wies demnach bei Krebsen des Rektums und des Urogennitalorgans geringe, bei denen des Gesichts und des Halses, der Mamma und der Extremitäten nur leichte Verminderung auf.

Es ist bekannt, dass Hämoglobin in roten Blutkörperchen für die Sauerstoffbindung eine grosse Rolle spielt. Autoren wie Kraus, Kossler u. Scholz stellten bei Anämie fest, dass, je mehr der Hämo- 
globingehalt des Bluts abnimmt, die Sauerstoffkapazität umso kleiner wird. Ausser 2 Ausnahmefällen (Fall 42 u. 45) wiesen meine Krebsfälle keine sehr schwere Anämie und auch keine starke Abnahme des Hämoglobingehalts im Blute auf. Jedoch wurde dabei, zwar nicht so genau wie bei Lund sga ard, doch im grossen und ganzen ein ungefähres Parallelgehen des Hämoglobingehalts oder der Erythrozytenzahl mit der Sauerstoffkapazität festgestellt. Ausnahmefälle mit entgegengesetztem Ergebnis wurden auch beobachtet. Bemerkt sei hier noch, dass unter den Magenkrebsen bei 8 das Fehlen freier Salzsäure im Magensaft nachgewiesen wurde und 5 davon stark verminderte Sauerstoffkapazität aufwiesen. Bei 3 unter 5 Fällen mit positiver Blutreaktion im Magensaft wurde auch erheblich verminderte Sauerstoffkapazität angetroffen. Diese starke Abnahme der Sauerstoffkapazität des venösen Bluts bei Magenkrebs dürfte auf einer besonderen Ernährungsstörung und damit verbundener starker Kachexie beruhen und deshalb mitunter als Gradmesser der Kachexie dienen können.

Odair a bemerkte bei Fällen mit Verstopfung, unter denen sich auch Rektumkrebse fanden, Azidosis verschiedenen Grades. Alle meine Fälle von Rektumkrebs hatten mehr oder weniger starke Neigung zur Verstopfung. Bei einem solchen inoperablen Fall (Fall 58) fand ich, 3 Tage nach Anlegung eines künstlichen Afters unter Lokalanästhesie, nur leichte Abnahme der Sauerstoffkapazität des venösen Bluts. Bei je einem Fall von Peniskrebs (Fall 64) und Oberkieferkrebs (Fall 67) wurde durch wiederholte Untersuchungen nach Pausen von mehreren Wochen oder Monaten festgestellt, dass die Verminderung der Sauerstoffkapazität mit Zunahme der Kachexie stärker wurde. Ferner wurde bei fast allen 4 Zungenkrebsen (Fall 73-76) verminderte Sauerstoffkapazität beobachtet. Nebenbei sei erwähnt, dass überhaupt die Sauerstoffkapazität venösen Bluts bei inoperablen Fällen von Krebs nach ein- oder halbjähriger Beobachtung deutliche Abnahme aufwies, bei Magenkrebs besonders stark, was zum Teil mit der Beschränkung der Nahrungsaufnahme in Zusammenhang zu stehen scheint.

Unter 7 von mir untersuchten Sarkomfällen fanden sich 4 Sarkome und 3 Lymphosarkome. Die Ergebnisse der Blutgasbestimmung bei diesen Fällen sind in Tabelle IV wiedergegeben. Bei allen diesen verhielt sich die Sauerstoffunsättigung des venösen Bluts fast normal. Bei 2 Fällen wurde sogar leichte Erhöhung beobachtet. Die Sauerstoffkapazität war bei allen meinen Sarkomfällen durchschnittlich 
etwas vermindert. Die prozentuelle Sauerstaffsättigung war bei Lymphosarkomen etwas kleiner.

Ferner wurde der Sauerstoff des venösen Bluts bei 38 Fällen chirurgischer Nierenerkrankungen, mit Ausschluss von Geschwülsten, bestimmt, unter denen es 27 Fälle chronischer Nierentuberkulose und wenige Fälle von Nierensteinkrankheit, Hydro- u. Pyonephrose, Nephritis chronica haematurica und Paranephritis usw. gab, von denen 25 zur Operation kamen (siehe Tabelle V). Bei 23 Fällen einseitiger Nierentuberkulose betrug die Sauerstoffunsättigung zwischen 13,24 und $5,25 \mathrm{ccm}$, durchschnittlich $7,97 \mathrm{ccm}$, die Sauerstoffkapazität zwischen 21,79 und $11,47 \mathrm{ccm}$, durchschnittlich $16,14 \mathrm{ccm}$ und die prozentuelle Sauerstoffsättigung durchschnittlich 50,6\%. Die Sauerstoffunsättigung war also dabei durchschnittlich etwas und die Sauerstoffkapazität nur ganz wenig vermindert. Die prozentuelle Sauerstoffsättigung blieb dabei normal. Es wurden aber auch Fälle beobachtet, bei denen die Sauerstoffunsättigung und -kapazität normal waren und sich die Nephrektomie mit Erfolg ausführen liess (z. B. Fall 93,100 u. 101). Solche Fälle kamen auch unter den sonstigen chirurgischen Nierenerkrankungen (mitunter bei einseitiger Erkrankung)

Tabelle V.

Untersuchungen bei chirurgischen Nierenerkrankungen.

\begin{tabular}{|c|c|c|c|c|c|c|c|}
\hline \multirow[b]{2}{*}{$\begin{array}{l}\text { Fall- } \\
\text { Nr. }\end{array}$} & \multirow[b]{2}{*}{$\begin{array}{c}\text { Name, } \\
\text { Alter } \\
\text { u. } \\
\text { Geschl. }\end{array}$} & \multirow[b]{2}{*}{$\underset{\text { (Seite) }}{\text { Klin. Diagn. }}$} & \multicolumn{3}{|c|}{$\begin{array}{l}\text { Sauerstoff- } \\
\text { bestimmung }\end{array}$} & \multirow{2}{*}{ 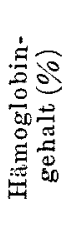 } & \multirow[b]{2}{*}{ Bemerkangen } \\
\hline & & & 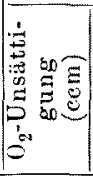 & 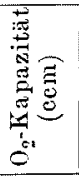 & 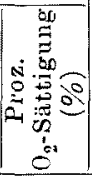 & & \\
\hline 93 & $\begin{array}{l}\text { T. T. } \\
26 \mathrm{~m}\end{array}$ & $\begin{array}{l}\text { Nierentbc. } \\
\text { (1) }\end{array}$ & 8,22 & 21,56 & 61,9 & 89 & Nephrektomie, Heilung \\
\hline 100 & $\begin{array}{l}\text { H. I. } \\
23 \mathrm{~m}\end{array}$ & $(r)$ & 7,56 & 16,13 & 53,1 & 81 & $"$ \\
\hline 101 & $\begin{array}{l}\text { B. H. } \\
30 \mathrm{~m}\end{array}$ & $(")$, & 8,11 & 19,58 & 58,6 & 90 & $"$ \\
\hline 117 & $\begin{array}{l}\text { H. O. } \\
32 \mathrm{w}\end{array}$ & $(\ddot{b d s})$ & 6,98 & 13,02 & 46,3 & - & Inoperabel \\
\hline 119 & $\begin{array}{l}\text { T.S. } \\
56 \mathrm{~m}\end{array}$ & $\begin{array}{l}\text { Nephritis chr. } \\
\text { haemat. (1) }\end{array}$ & 8,34 & 13,93 & 40,1 & 61 & Dekapsulation \\
\hline 125 & $\begin{array}{l}Y . S . \\
44 \mathrm{~m}\end{array}$ & $\begin{array}{l}\text { Nierenstein } \\
\text { (bds) }\end{array}$ & 7,07 & 13,45 & 47,4 & - & $\begin{array}{l}\text { Nephro- u. Uretero- } \\
\text { lithotomie }\end{array}$ \\
\hline
\end{tabular}


vor. Hier wurden aber auch Ausnahmefälle mit stark verminderter Sauerstoffkapazität beobachtet, wie bei einem Fall nicht tuberkulöser Pyonephrosis (Fall 121), den man erfolgreich nephrektomieren konnte.

Bei beiderseitiger Nierentuberkulose (z. B. Fall 117) hatten die Sauerstoffunsättigung und -kapazität mehr oder weniger deutlich abgenommen, und die prozentuelle Sanerstoffsättigung lag dabei etwas unter der Norm. Dasselbe gilt für beiderseitige Nierensteinkrankheit (z. B. Fall 125). Bei einem Fall von Nephritis chronica haematurica (Fall 119), der wegen einseitiger starker Renalblutung dekapsuliert wurde, liess sich deutlich verminderte Sauerstoffkapazität sowie prozentuelle Sauerstoffsättigung beobachten, was zum Teil auf starkem Blutverlust beruhen dürfte.

Die Sauerstoffkapazität zeigte bei den Fällen chirurgischer Nierenerkrankungen, besonders chronischer Nierentuberkulose, keinen Parallelismus mit dem Funktionszustand der Nieren, d.h. der Ausscheidung von Phenolsulfophthalein, auch bei doppelseitigen Erkrankungen nicht. Vielmehr wurde dabei Abhängigkeit der Sauerstoffkapazität des Bluts vom allgemeinen Ernährungszustand bemerkt. Auch wurde dabei durch wiederholte Untersuchungen festgestellt, dass die verminderte Sauerstoff kapazität des Bluts nach Nephrektomie oft zugleich mit der Erholung des Körpers allmählich wieder zum normalen Durchschnitt anstieg.

Meine Fälle akuter eitriger Infektion (siehe Tabelle VI) betrugen im ganzen 31, nämlich 15 Appendicitis acuta, 10 Perforationsperitonitis, meistens appendikulären oder seltener sonstigen Ursprungs und 6 akute eitrige Infektion (Myositis, Mastitis usw.). Als Fälle chronischer Infektion wurden 7 chirurgische Tuberkulosen, vor allem der Lymphdrüse, und 1 Hodengumma untersucht. Ausserdem untersuchte ich als Kontrolle im ganzen 16 Fälle sonstiger chirurgischer Erkrankungen, unter denen sich Ileus, Hernia, Struma und Analpolypen fanden.

Alle 7 Fälle von Appendicitis acuta (Intervall), bei denen ich die Bestimmung unter den oben erwähnten Bedingungen ausgeführt hatte, zeigten annähernd normale Werte der Sauerstoffunsättigung, -kapazität und prozentuellen Sauerstoffsättigung. Bei den sonstigen 8 Fällen von früh operierter Appendicitis acuta und bei den Fällen von Perforationsperitonitis, Darmverschluss sowie Herniainkarzeration wurden bei der Bestimmung die oben erwähnten Bedingungen wegen der Notoperation nicht immer erfüllt. Bei diesen früh operierten Appen diz itisfällen war der Durchschnittswert der Sauerstoffunsättigung. 
Tabelle VI.

Untersuchungen bei Infektionen.

\begin{tabular}{|c|c|c|c|c|c|c|c|}
\hline \multirow[b]{2}{*}{$\begin{array}{l}\text { Fall- } \\
\mathrm{Nr} \text {. }\end{array}$} & \multirow[b]{2}{*}{$\begin{array}{c}\text { Name, } \\
\text { Alter } \\
\text { u. } \\
\text { Gesehl. }\end{array}$} & \multirow[b]{2}{*}{$\begin{array}{l}\text { Klin. } \\
\text { Diagnose }\end{array}$} & \multicolumn{3}{|c|}{$\begin{array}{l}\text { Sauerstoff- } \\
\text { bestimmung }\end{array}$} & \multirow[b]{2}{*}{ 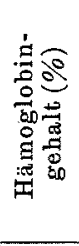 } & \multirow[b]{2}{*}{ Bemerkungen } \\
\hline & & & 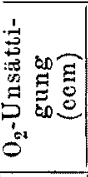 & 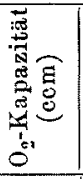 & 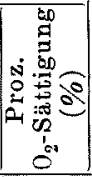 & & \\
\hline 135 & $\begin{array}{l}\text { S. S. } \\
23 \mathrm{~m}\end{array}$ & $\begin{array}{l}\text { Appendicitis } \\
\text { acuta }\end{array}$ & 8,12 & 18,33 & 55,7 & 94 & Appendektomie \\
\hline 140 & $\begin{array}{l}\text { H. S. } \\
43 \mathrm{w}\end{array}$ & $\begin{array}{l}\text { Appendicitis } \\
\text { gangraenosa }\end{array}$ & 5,79 & 15,34 & 62,3 & 87 & ", \\
\hline 150 & $\begin{array}{l}\text { K. S. } \\
12 \mathrm{w}\end{array}$ & $\begin{array}{l}\text { Perforations- } \\
\text { peritonitis }\end{array}$ & 6,79 & 14,56 & 53,3 & 92 & $\begin{array}{l}\text { Appendektomie } \\
\text { Exitus letalis }\end{array}$ \\
\hline 152 & $\begin{array}{l}\text { H. S. } \\
40 \mathrm{~m}\end{array}$ & $"$ & 8,39 & 13,59 & 38,3 & 62 & $\begin{array}{l}\text { Notop. mit G. E. post. } \\
\text { Exitus letalis }\end{array}$ \\
\hline 175 & $\begin{array}{l}\text { S. E. } \\
38 \mathrm{~m}\end{array}$ & $\begin{array}{l}\text { Myositis acuta } \\
\text { purulenta }\end{array}$ & 5,12 & 16,43 & 68,8 & 71 & Inzision \\
\hline 166 & $\begin{array}{l}\text { J.A. } \\
25 \mathrm{~m}\end{array}$ & $\begin{array}{l}\text { Bds. Neben- } \\
\text { hodentbe. }\end{array}$ & 7,23 & 16,29 & 55,6 & - & Kastration \\
\hline 168 & $\begin{array}{l}\text { N. K. } \\
43 \mathrm{~m}\end{array}$ & Ileozoekaltbc. & 9,29 & 12,48 & 25,4 & 88 & Ileokolostomie \\
\hline
\end{tabular}

und -kapazität etwas niedriger als bei Intervallfällen. Bei Perforationsperitonitis hatte die Sauerstoffkapazität im allgemeinen mehr oder weniger deutlich abgenommen, besonders bei Fällen mit ungünstigem Ausgang. Der Durchschnitt der prozentuellen Sauerstoffsättigung betrug bei Perforationsperitonitis jedoch durchnittlich $50,5 \%$. Bei meinen sonstigen akuten Infektionen war die Sauerstoffunsättigung normal oder etwas vermindert, und die Sauerstoffkapazität zeigte leichte Abnahme. Bei meinen chirurgischen Tuberkulosen wurden meistens annähernd normale Sauerstoffsättigung und -kapazität, aber auch Ausnahmen, beobachtet. Bei einem fortgeschrittenen Fall von Tleozoekaltuberkulose (Fall 168) fand ich normale Sauerstoffunsättigung, aber erheblich verminderte Sauerstoffkapazität sowie prozentuelle Sauerstoffsättigung. 


\section{Schluss.}

1. Ich bestimmte den Sauerstoff (die Sauerstoffunsättigung, -kapazität und prozentulle Sauerstoffsättigung)venösen Bluts mit dem Barcroftschen Differentialmanometer (Makroapparat) bei Gesunden, bösartigen Geschwülsten, vor allem auch Krebsen und sonstigen chirurgischen Erkrankungen, einschliesslich chirurgischer Nierenleiden sowie akuter und chronischer Infektionen. Die Gesamtzahl der untersuchten Fälle machte 183 (209 Untersuchungen) aus. Darunter fanden sich 39 Gesunde, 51 maligne Tumoren, 38 chirurgische Nierenerkrankungen, mit Ausschluss der Geschwülste, und 55 akute und chronische Infektionen sowie sonstige chirurgische Erkrankungen. Dabei wurden als Regel gleichzeitig auch Pulsfrequenz, Atmungszahl, Körpertemperatur, Blutkörperchenzahl und Hämoglobingehalt untersucht. Bei Magenkrebs wurde daneben auch der Magensaft untersucht und bei chirurgischen Nierenerkrankungen die Nierenfunktion geprüft.

2. Bei meinen 39 Gesunden betrug die Sauerstoffunsättigung venösen Bluts zwischen $15,86 \mathrm{ccm}$ und $6,27 \mathrm{ccm}$, durchschnittlich 9,08 ccm in $100 \mathrm{ccm}$ Blut bei $0^{\circ} \mathrm{C}, 760 \mathrm{~mm} \mathrm{Hg}$, die Sauerstoffkapazität zwischen $22,44 \mathrm{ccm}$ und $16,40 \mathrm{ccm}$, durchschnittlich $19,59 \mathrm{ccm}$ in 100 ccm Blut bei $0^{\circ} \mathrm{C}, 760 \mathrm{~mm} \mathrm{Hg}$. Die prozentuelle Sauerstoffsättigung betrug dann durchschnittlich $53,6 \%$. Die Sauerstoffunsättigung und -kapazität waren bei Männern etwas höher als bei Frauen.

3. Bei meinen Fällen maligner Geschwülste, vor allem bei Krebsen, war die Sauerstoffunsättigung des venösen Bluts fast normal oder nur etwa erhöht, dagegen die Sauerstoffkapazität fast immer mehr oder weniger stark vermindert. Dies war besonders bei Magenkrebs mit Kachexie der Fall. Die prozentuelle Sauerstoffsättigung fand sich dabei stärker vermindert.

4. Bei den 38 Fällen chirurgischer Nierenleiden, ausschliesslich der Geschwülste, wovon die Nierentuberkulose die Hauptzahl ausmachte, zeigten die meisten mit Erfolg operierten Fälle und diejenigen, die bei sonstigen Prüfungsmethoden auch gute Nierenfunktion aufgewiesen hatten, annähernd normale oder etwas verminderte Sauerstoffunsättigung, -kapazität sowie prozentuelle Sauerstoffsättigung. Bei beiderseitiger Erkrankung und bei inoperablen Fällen wurde mitunter erheblich verminderte Sauerstoff kapazität bemerkt. Bei allen diesen zeigte die Sauerstoff kapazität nicht immer Parallelismus mit der Ausscheidung des Phenolsulphophthaleins, schien aber 
zum Teil von der allgemeinen Ernährung des Körpers abhängig zu sein.

5. Bei meinen Fällen akuter eitriger Infektion, d.h. bei Appendicitis acuta, bei Perforationsperitonitis appendikulären und sonstigen Ursprungs, sowie bei akuten Eiterungen wurden normale oder etwas verminderte Sauerstoffunsättigung, -kapazität und prozentuelle Sauerstoffsättigung beobachtet. Dasselbe traf bei meinen Fällen mit chronischer Infektion, besonders chirurgischer Tuberkulose, zu. Dagegen wurde bei fortgeschrittenen Fällen akut-eitriger oder tuberkulöser Infektion oft mehr oder weniger starke Abnahme der Sauerstoffkapazität des venösen Bluts beobachtet.

6. Durch die in gewissen Pausen wiederholt ausgeführten Bestimmungen konnte ich feststellen, dass die Sauerstoffkapazität venösen Bluts bei malignen Geschwülsten, vor allem bei Krebsen, oft mit zunehmender Kachexie sinkt. Auch wurde mitunter beobachtet, dass die Sauerstoffkapazität nach erfolgreicher Operation, z. B. bei chirurgischem Nierenleiden, mit der Zeit ansteigt und schliesslich einen annähernd normalen Wert erreicht.

7. Nach Obigem darf man annehmen, dass die Bestimmung des Sauerstoffs venösen Blutes, besonders der Sauerstoffkapazität, mitunter einen wertvollen Aufschluss über den kachektischen Zustand bei chirurgischen Erkrankungen, besonders bei malignen Geschwiilsten, und hier vor allem bei Karzinomen, gibt.

\section{Literature.}

(1) B a r croft, J., The respiratory function of the blood. Cambridge 1914, 290 bis 314.

(2) Burrows, M. T., Journ. Canc. Research, 1921, 6, 131-138. 566.

(3) Gorasch, W. A., Urologia, 1924, 3, 22-24. Ref: Ztrlorg. ges. Chir. 1925, 31,

(4) Haden, R.T. u. T. G. O rr, Journ. Exp. Med., 1927, 46, 709-713.

(5) Haldane, J. u. J. L. S m it h, Journ. Physiol., 1900, 25, 331-343.

(6) Harrop, G.A., Journ. Exp. Med., 1919, 30, 241-257.

(7) Hoff ma n n, P., Journ. Physiol., 1913-14, 47, 272-274.

(8) Hürter, Dtsch. Arch. kl. Med., 1912, 108, 1-34.

(9) I iz u ka, N., Igakn Chuo Zasshi, 1924, 22, 1-14.

(10) Ka to, T., Nippon Naika Gakkai Zasshi, 1922, 9, 705-809.

(11) Ki m u ra, N. u. M. Y a m a d a, Shinkeigaku Zasshi, 1928, 29, 286-288.

(12) Kraus, Fr., Kossler u. W. Scholz, Arch. exp. Path. u. Pharm., 1899, 42, 323-355.

(13) Lundsga ard, C., Journ. Biol. Chem., 1918, 33, $133-144$.

(14) Lundsgaard, C., Journ. Exp. Med., 1919, 30, 147-158. 
(15) Luttinge r, P., Cancer, 1925, 3, 28-37. Ref: Ztrolorg. ges. Chir., 1927, 38, 552.

(16) Mento n, M. L., Journ. Canc. Research, 1917, 2, 179-211.

(17) Moore, B. u. F. P. Wils o n, Bioch. Journ., 1906, 1, 297-327.

(18) Odaira, T., Tohoku Journ. Exp. Med., 1921, 2, 570-598.

(19) Odair a, T., Tohokn Igaku Zasshi, 1922, 6, 209-215.

(20) O daira, T., Nippon Naika Gakkai Zasshi, 1925, 13, 295-316.

(21) Porges, 0. u. A. Leim dörfer, Ztschr. kl. Med., 1913, 77, 464-470.

(22) Posharskaja, Russki Wratsch, 1913, 12, 529-531 u. 562-565. Ref: Ztrlorg. ges. Chir. 1913. 2, 785-786.

(23) Roffo, A. H. u. L. M. Correa, Prensa med. Argent., 1924, 11, 502-508. Ref: Ztrlorg. ges. Chir. 1926. 33, 406-407.

(24) Sannié, C. u. Ed. P e y re, Bull. l'Assoc. Franç. pour l'Etude du Cancer, 1926, 15, 199-212.

(25) Se hrt, E., Dtsch. med. Wochenschr., 1929, 1666-1667, u. Ztschr. Krebsforsch. 1930, 30, 260-270. 44, 269 .

(26) Se jh a r, J., Cas. lek. cesk., 1928 (II), 1089-1092. Ref : Ztrlorg. ges. Chir. 1929,

(27) Stadie, W. C., Journ. Exp. Med., 1919, 30, 215-240.

(28) Straub, H., Mechnik der Blutgasanalyse nach Barcroft; Emil Abderhald e n's Handbuch der biologischen Arbeitsmethoden. Berlin u. Wien 1926, Abt. IV, Teil 10, 213-234.

(29) Suganuma, S., Tokyo Igakkai Zasshi, 1920, 34, 381-401.

(30) S ugawara, T. Tohoku Igaku Zasshi, 1919, 4, 264-302.

(31) T a naka, A., Kaigun Gnnikai Zasshi, 1922, Nr. 38, 275-292.

(32) Un o, K., Nippon Geka Gakkai Zasshi, 1924, 25, 45-76.

(33) Van a, A., Mitteilg. Grenzgeb. Med. u. Chir., 1927, 40, 514-541.

(34) We iss, S., S. Sümegi u. L. v. Udvardy, Kl. Wochenschr, 1928, 1178-1182.

(35) Yamauchi, M., Chiba Igakkai Zasshi, 1928, 6, 231-232. 